International Mathematical Forum, Vol. 8, 2013, no. 38, 1863 - 1872

HIKARI Ltd, www.m-hikari.com

http://dx.doi.org/10.12988/imf.2013.310198

\title{
On Open Domination and Domination in Signed Graphs
}

\author{
K. A. Germina \\ School of Mathematical and Physical Sciences \\ Central University of Kerala \\ Kasaragod - 671328, Kerala, India \\ srgerminaka@gmail.com \\ P. K. Ashraf \\ Department of Mathematics \\ Sir Syed College \\ Taliparamba, Kannur, Kerala- 670142 \\ ashrafkalanthod@gmail.com
}

Copyright (C) 2013 K. A. Germina and P. K. Ashraf. This is an open access article distributed under the Creative Commons Attribution License, which permits unrestricted use, distribution, and reproduction in any medium, provided the original work is properly cited.

\begin{abstract}
A graph whose edges are labeled either as positive or negative is called a signed graph. Motivated from the seminal paper of B. D. Acharya on domination in signed graphs, we in this article, provide answers to some of the problems in that paper and define open domination for a signed graph and its open domination number. We analyze this notion for certain classes of signed graphs and also give exact values for their open domination numbers. We also discuss about certain bounds for the open domination number of a signed graph.
\end{abstract}

Mathematics Subject Classification: 05C10, 05C22

Keywords: signed graph, domination, open domination, open domination number 


\section{Introduction}

All graphs in this article are simple. For all definitions in (unsigned) graph theory used here, unless otherwise mentioned, reader may refer to [5] and for the terminology and definitions in the theory of dominations for simple graphs, we refer the reader to $[2,7]$. Signed graphs (also called sigraphs) are graphs with positive or negative labels on the edges. Formally, a sigraph is an ordered pair $\Sigma=(G, \sigma)$ where $G=(V, E)$ is a graph called the underlying graph of $\Sigma$ and $\sigma: E \rightarrow\{+1,-1\}$ called a signing, is a function (also called a signature) from the edge set $E$ of $G$ into the set $\{+1,-1\}$. For more details on theory and applications of signed graphs, one may refer to $[3,4,8,9]$.

A signed graph is all-positive (respectively, all-negative) if all of its edges are positive (negative); further, it is said to be homogeneous if it is either all-positive or all-negative and heterogeneous otherwise. $+G$ denotes an allpositive graph and $+K_{n}$, an all-positive complete graph. Similarly $-K_{n}$ represents an all-negative complete graph. Note that a graph can be considered to be a homogeneous signed graph. A signed graph $\Sigma$ is said to be balanced or cycle balanced if all of its cycles are positive, where the sign of a cycle is the product of the signs of its edges. We use $N(u)$ to denote the open neighbourhood of a vertex $u$ in a graph .

We denote by $P_{n}^{(r)}$, where $0 \leq r \leq n-1$, signed paths of order $n$ and size $n-1$ with $r$ negative edges. Also $C_{n}^{(r)}$, for $0 \leq r \leq n$, denotes signed cycles with $r$ negative edges. If $\zeta: V \rightarrow\{+1,-1\}$ is a function, called a switching function, then switching of the signed graph $\Sigma=(G, \sigma)$ by $\zeta$ means changing $\sigma$ to $\sigma^{\zeta}$ defined by:

$$
\sigma^{\zeta}(u v)=\zeta(u) \sigma(u v) \zeta(v)
$$

The switched graph, denoted by $\Sigma^{\zeta}$, is the signed graph $\Sigma^{\zeta}=\left(G, \sigma^{\zeta}\right)$. We call two signed graphs $\Sigma_{1}=\left(G, \sigma_{1}\right)$ and $\Sigma_{2}=\left(G, \sigma_{2}\right)$ switching equivalent, if there exists a switching function $\zeta: V \rightarrow\{+1,-1\}$ such that $\Sigma_{1}=\Sigma_{2}^{\zeta}$.

The following important result is often used in signed graph theory.

Lemma 1.1 ([8]). A signed graph is balanced if and only if it can be switched to an all-positive signed graph.

\section{Domination in Signed Graphs}

B D Acharya initiated the discussion of domination theory for signed graphs in [1] as follows.

Definition 2.1. Let $\Sigma=(V, E, \sigma)$ be a signed graph. A subset $D \subseteq V$ of vertices of $\Sigma$ is a dominating set of $\Sigma$, if there exists a marking $\mu: V \rightarrow$ $\{+1,-1\}$ of $\Sigma$ such that every vertex $u$ of $\Sigma$ is either in $D$ or whenever $u \in$ $V \backslash D, N(u) \cap D \neq \emptyset$ and $\sigma(u v)=\mu(u) \mu(v)$ for every $v \in N(u) \cap D$. 
A dominating set $D$ of $\Sigma$ is called a minimal dominating set if no proper subset of $D$ becomes a dominating set. A minimum dominating set is a minimal dominating set with least cardinality. This least number is called domination number of $\Sigma$ and is denoted by $\gamma(\Sigma)$. Before we proceed further, we note that the following is an important result in [1] which will be used in the sequel.

Theorem 2.2 ([1]). Domination is invariant under switching.

In view of the Theorem 2.2 and the Lemma 1.1, it is interesting to see the discussion of domination in signed graph is more relevant when the signed graph is unbalanced. In the following results we address some of the problems found in [1] associated with the domination number and domination of a signed graph. We denote the set of all dominating set by $\mathcal{D}_{\Sigma}$. Clearly

$$
\mathcal{D}_{\Sigma} \subseteq \mathcal{D}_{G}
$$

In [1], a problem is given to determine the signed graphs such that $\mathcal{D}_{\Sigma}=\mathcal{D}_{G}$. In view of Theorem 2.2 and Lemma 1.1, all balanced signed graphs $\Sigma$ will satisfy this condition which is proved in [1]. In this regard, we have the following result to say.

Theorem 2.3. If $D \in \mathcal{D}_{G}$ is such that $N(u) \cap N(v)=\emptyset$ for all $u, v \in D$, then $D \in \mathcal{D}_{\Sigma}$.

Proof. Let $D \in \mathcal{D}_{G}$ satisfies the property given in the statement of the theorem. If $D=V$ the proof follows easily from the definition. If not, take the marking $\mu$ such that $\mu(u)=+1$ for all the vertices in $u \in D$ and since $N(u) \cap N(v)=\emptyset$ for all $u, v \in D$, we set $\mu$ in $V \backslash D$ as follows. For a vertex $w \in V \backslash D$, we take $\mu(w)=\sigma(u w)$ whenever $u \in N(w) \cap D$. Such a marking will satisfy definition 2.1. Hence $D \in \mathcal{D}_{\Sigma}$.

The dominating set $D$ in Theorem 2.3, is called a perfect dominating set. Indeed perfect dominating sets in the case of signed graphs will play a crucial role which we shall see in the subsequent sections.

\subsection{Domination number of a signed graph}

Now we give exact values for the domination number of certain classes of signed graphs. We use the following result given in [2] to find the domination number of signed graph built on complete bipartite graph $K_{m, n}$.

Lemma $2.4([2]) \cdot \gamma\left(K_{m, n}\right)=\min \{m, n, 2\}$.

Theorem 2.5. $\gamma\left(K_{m, n}^{(r)}\right)=\gamma\left(K_{m, n}\right)=\min \{m, n, 2\}$. 
Proof. If $\min \{m, n\}=1, K_{m, n}^{(r)}$ is a signed star which is balanced and the result follows. If $\min \{m, n\} \geq 2$, take a minimum dominating set $D \in \mathcal{D}_{K m, n}$ whose cardinality is 2 (see Lemma 2.4) with its members $u$ and $v$ in different parts. We claim that $N(u) \cap N(v)=\emptyset$. If not, let $w \in N(u) \cap N(v)$ which implies that $w$ belongs to both parts which is contradiction to the bipartition. Then the remaining part follows from Theorem 2.3.

Theorem 2.6. $\gamma\left(C_{n}^{(r)}\right)=\gamma\left(C_{n}\right)=\left\lceil\frac{n}{3}\right\rceil$ for all $r$ such that $0 \leq r \leq n$.

Proof. If $r$ is even, as $C_{n}^{(r)}$ is balanced, the result follows from Theorem 2.2 and Lemma 1.1. In the other case, in light of Theorem 2.2, it is enough to prove the result for the case $r=1$. Without loss of generality, let $C_{n}^{(1)}: v_{1} v_{2} \ldots v_{n} v_{1}$ be the cycle with $v_{2} v_{3}$ being negative. Let $D$ be that minimum dominating set of $C_{n}$ constructed as follows. Taking $v_{1}$ as the first element in $D$, move cyclically around the cycle in such a way that we select every fourth vertex of the cycle in order until we selected $\left\lceil\frac{n}{3}\right\rceil$ vertices to be in $D$.(Note that we only have to select $\left\lceil\frac{n}{3}\right\rceil$ vertices in $D$ since $|D|=\gamma\left(C_{n}\right)=\left\lceil\frac{n}{3}\right\rceil($ cf. [2] $)$ ). Now we argue that this $D$ will be a minimum dominating set for $C_{n}^{(1)}$. For, the adopted method of selection described above brings together $v_{2}$ and $v_{3}$ in $V \backslash D$. Thus the only negative edge $v_{2} v_{3}$ will create no problem for the marking and the all-positive marking will solve the issue.

In Theorem 2.5 and Theorem 2.6, we have $\gamma(\Sigma)=\gamma(G)$. In Fig. 1, we give a signed graph $\Sigma$ for which $\gamma(\Sigma)>\gamma(G)$.

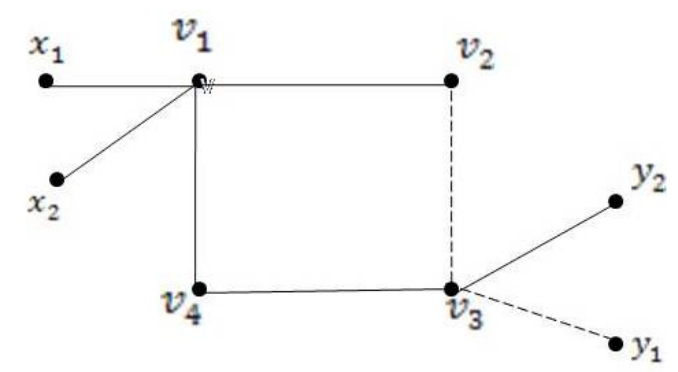

Figure 1: $\gamma(\Sigma)>\gamma(G)$

The only minimum dominating set for the underlying graph $G$ in the above figure is $\left\{v_{1}, v_{3}\right\}$ because we require them to dominate $x_{1}, x_{2}$ and $y_{1}, y_{2}$ which are respectively connected only to $v_{1}$ and $v_{3}$. But this minimum dominating set is not even a dominating set for $\Sigma$ because no marking is possible satisfying the definition of dominating set. Also no other set of cardinality 2 will be a dominating set for $\Sigma$. To see this, one can easily verify that sets of cardinality 2 other than $\left\{v_{1}, v_{3}\right\}$ will not be dominating sets for $G$ and hence for $\Sigma$, by 
Equation (2).

The above example gives raise to a more general result in the following manner. Note that $\gamma\left(C_{4}^{(1)}\right)=\gamma\left(C_{4}\right)$, but when $C_{4}^{(1)}$ gets 'bridged' in a particular way as described below it provides the following interesting result. The bridging operation of $C_{4}^{(1)}$ with two signed graphs $\Sigma_{1}=\left(G_{1}, \sigma_{1}\right)$ and $\Sigma_{2}=\left(G_{2}, \sigma_{2}\right)$ where both $G_{1}$ and $G_{2}$ are two non-complete graphs having at least one full degree vertex for each and satisfy $\Delta\left(G_{1}\right) \geq 2, \Delta\left(G_{2}\right) \geq 2$, is defined by identifying one full degree vertex in each of $\Sigma_{1}$ and $\Sigma_{2}$ with two non-adjacent vertices of $C_{4}^{(1)}$. Let us denote the resultant graph obtained after this operation by $\Sigma_{1} \diamond \Sigma_{2}$. Note that for the underlying graphs $G_{1}$ and $G_{2}$, in $G_{1} \diamond G_{2}$, we take the bridging cycle as $C_{4}$.

Theorem 2.7. $\gamma\left(\Sigma_{1} \diamond \Sigma_{2}\right)>\gamma\left(G_{1} \diamond G_{2}\right)$

Proof. Let $u$ and $v$ be the non-adjacent vertices of $C_{4}^{(1)}$ where we attach the graphs $G_{1}$ and $G_{2}$, so that the full degree vertices of $G_{1}$ and $G_{2}$ are identified with one of $u$ or $v$. Now assuming that the full degree vertex of $G_{1}$ is identified with $u$ and that of $G_{2}$ is identified with $v$. Then $d_{\left(G_{1} \diamond G_{2}\right)}(u)=\left|V\left(G_{1}\right)\right|-1+2=$ $\left|V\left(G_{1}\right)\right|+1$ and $d_{\left(G_{1} \diamond G_{2}\right)}(v)=\left|V\left(G_{2}\right)\right|+1$, then our claim is that $\{u, v\}$ is the only minimum dominating set for $G_{1} \diamond G_{2}$ but not a even a dominating set for $\Sigma_{1} \diamond \Sigma_{2}$. For, $u$ dominates all the vertices of $G_{1}$ and the remaining vertices $w$ and $z$ of the cycle. $v$ takes care of the vertices in $G_{2}$. Moreover any dominating set of cardinality two require both $u$ and $v$ as they are needed to dominate the vertices of $G_{1}$ and $G_{2}$ respectively. But in the case of the signed graph $\Sigma_{1} \diamond \Sigma_{2}$, no marking is possible for this set $\{u, v\}$ as per definition 2.1. The reasons for this are better illustrated in Fig. 2 and Fig. 3 where $w$ and $z$ are the remaining vertices of $C_{4}^{(1)}$. Indeed, $\gamma\left(\Sigma_{1} \diamond \Sigma_{2}\right)=1+\gamma\left(G_{1} \diamond G_{2}\right)$ as $\{u, v, w\}$ or $\{u, v, z\}$ will be a minimum dominating set for $\Sigma_{1} \diamond \Sigma_{2}$.

The above example and the fact that $\gamma(G) \leq \gamma(\Sigma) \leq|V(G)|$ give raise to the following problems.

Problem 2.8. If $k$ is a positive integer such that $\gamma(G) \leq k \leq|V(G)|$, which is that signed graph $\Sigma=(G, \sigma)$ such that $\gamma(\Sigma)=k$ ?

Problem 2.9. If $k$ is a non-negative integer such that $0 \leq k \leq|V(G)|-\gamma(G)$, which is that signed graph $\Sigma=(G, \sigma)$ such that $\gamma(G)+k=\gamma(\Sigma)$ ?

\section{Open Domination in Signed graphs}

We define $D \subseteq V$ as an open dominating set (also called total dominating set) of the signed graph $\Sigma=(V, E, \sigma)$, if every vertex of $\Sigma$ is adjacent to at least 


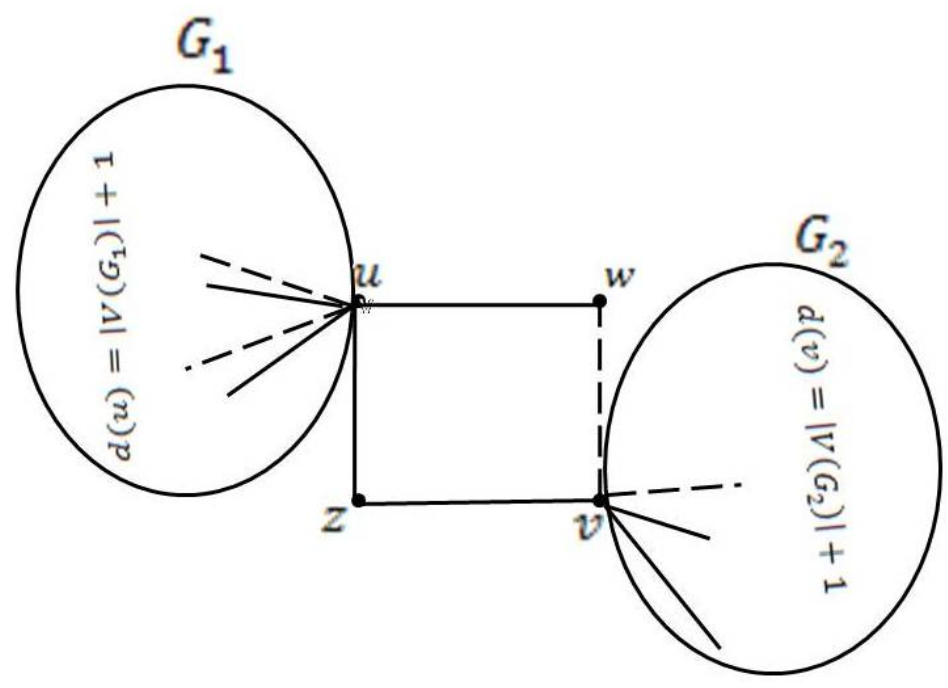

Figure 2: $\gamma\left(\Sigma_{1} \diamond \Sigma_{2}\right)>\gamma\left(G_{1} \diamond G_{2}\right)$

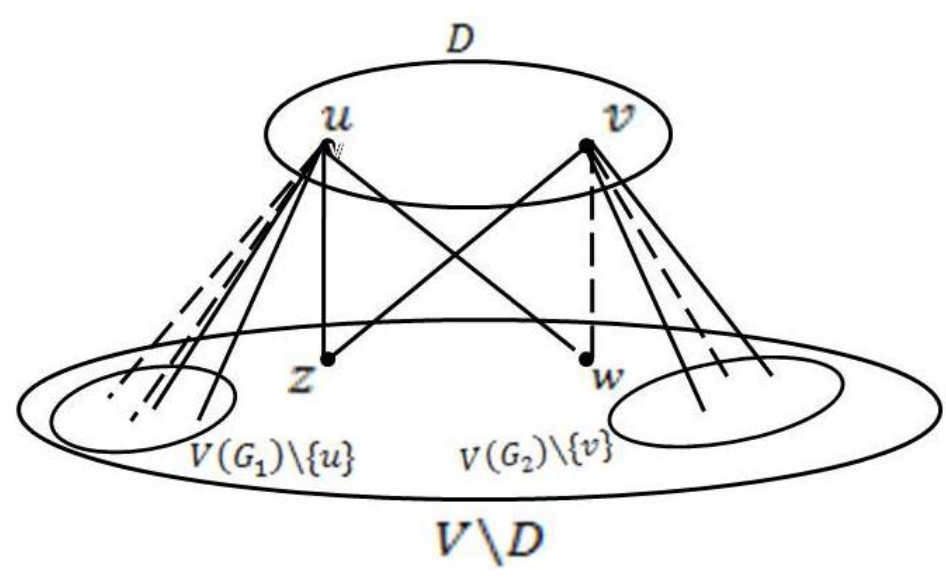

Figure 3: Illustration for Theorem 2.7

one vertex of $D$ and if there exists a marking $\mu: V \rightarrow\{+1,-1\}$ such that $\sigma(u v)=\mu(u) \mu(v)$ for every adjacent $u \in D$ and $v \in V \backslash D$.

As each vertex in an open dominating set $D$ is adjacent with at least one other vertex in $D$ itself (which is not the case with a dominating set), the kernel of a signed graph $\Sigma$ in the case of open domination is always empty. Recall that kernel is the independent dominating set.

It is easy to see that the definition takes care of the case of ordinary graph $G$ which can be thought of as an all-positive signed graph $+G$. In this case the marking $\mu: V(+G) \rightarrow\{+1,-1\}$ can be taken as the $\mu(v)=+1$ for all $v \in V(+G)$. 
Moreover, $\Sigma$ must be isolate-free since in the case of open domination no vertex is allowed to dominate itself.

We denote the set of all open dominating sets of a signed graph $\Sigma$ by $\mathcal{D}_{\Sigma}^{o}$. The following observvation is an easy consequence of the definition.

\section{Observation 3.1.}

$$
\mathcal{D}_{\Sigma}^{o} \subseteq \mathcal{D}_{G}^{o}
$$

Equation (3) raises the following natural open question.

Problem 3.2. Determine all signed graphs $\Sigma$ such that

$$
\mathcal{D}_{\Sigma}^{o}=\mathcal{D}_{G}^{o}
$$

Fig. 4 is a signed graph $\Sigma$ (a signed wheel) that does not satisfy Equation (4). Here $\left\{v_{2}, v_{5}\right\}$ is an open dominating set for the underlying wheel but not for the signed wheel.

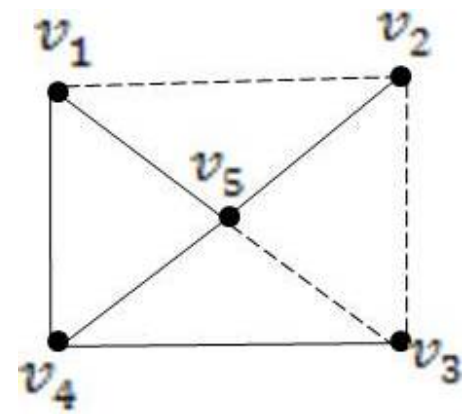

Figure 4: Signed wheel

Lemma 3.3. Open domination is switching invariant. i.e., $\mathcal{D}_{\Sigma}^{o}=\mathcal{D}_{\Sigma \zeta}^{o}$.

Proof. Let $D \in \mathcal{D}_{\Sigma}^{o}$. Then there exists a marking $\mu$ such that $\sigma(u v)=$ $\mu(u) \mu(v)$. To prove that $D \in \mathcal{D}_{\Sigma \zeta}^{o}$, we note that from Equation (1), $\sigma^{\zeta}(u v)=$ $\zeta(u) \mu(u) \mu(v) \zeta(v)$. Then setting the function $\mu^{\zeta}=\zeta \mu$ we get $\sigma^{\zeta}(u v)=\mu^{\zeta}(u) \mu^{\zeta}(v)$, showing that $D \in \mathcal{D}_{\Sigma^{\zeta}}^{o}$. Converse follows easily from the fact that $\left(\Sigma^{\zeta}\right)^{\zeta}=$ $\Sigma$.

In view of the Lemma 3.3, we have the following important proposition which makes the discussion of open domination in the case of unbalanced signed graphs very interesting.

Proposition 3.4. In the case of balanced signed graphs $\Sigma, \mathcal{D}_{\Sigma}^{o}=\mathcal{D}_{G}^{o}$.

Proof. As $\Sigma=(G, \sigma)$ is balanced, there exists a switching function $\zeta$ such that $\Sigma^{\zeta}=+G$. Then the result follows from Lemma 3.3 and Lemma 1.1. 
Theorem 3.5. If $D \in \mathcal{D}_{G}^{o}$ is such that $N(u) \cap N(v)=\emptyset$ for all $u, v \in D$ then $D \in \mathcal{D}_{\Sigma}^{o}$.

Proof. In view of Equation (3) it is enough to prove here that $\mathcal{D}_{G}^{o} \subseteq \mathcal{D}_{\Sigma}^{o}$ for those $D$ satisfying the conditions in the theorem. Let $D \in \mathcal{D}_{G}^{o}$. Mark the vertices in $D$ to be positive. Since $N(u) \cap N(v)=\emptyset$, we have the freedom to define the marking $\mu$ as follows. For a vertex $w \in V \backslash D$, we take $\mu(w)=+1$ or -1 according as $\sigma(u w)=+1$ or -1 . Such a marking will satisfy definition 2.1. Hence $D \in \mathcal{D}_{\Sigma}$.

\subsection{Open domination number}

The minimum cardinality of an open dominating set is defined as the open domination number of $\Sigma$ denoted by $\gamma_{t}(\Sigma)$. An open dominating set of cardinality $\gamma_{t}(\Sigma)$ is a minimum open dominating set for $\Sigma$. Since every open dominating set of a signed graph $\Sigma$ of order $n$ has at least two elements, $2 \leq \gamma_{t}(\Sigma) \leq n$. The inequality is sharp as the lower bound can be attained by a signed star and the upper bound is attained by the signed graphs built on the underlying graph $G=\frac{n}{2} K_{2}$ when $n$ is even.

Remark 3.6. Since every open dominating set in $\Sigma$ is also a dominating set

$$
\gamma(\Sigma) \leq \gamma_{t}(\Sigma)
$$

Towards showing that Inequality (5) can be strict but also can be an equality, we have $\gamma\left(C_{6}^{(1)}\right)=2$ and $\gamma_{t}\left(C_{6}^{(1)}\right)=4$. Also $\gamma\left(C_{4}^{(1)}\right)=2=\gamma_{t}\left(C_{4}^{(1)}\right)$.

Remark 3.7. For a signed graph $\Sigma$, its vertex set $V(\Sigma)$ is trivially an open dominating set of $\Sigma$. Further since $\mathcal{D}_{\Sigma}^{o} \subseteq \mathcal{D}_{G}^{o}$, we must have in general

$$
\gamma_{t}(G) \leq \gamma_{t}(\Sigma)
$$

In Fig. 5, we have a signed graph $\Sigma$ built on the complete graph $K_{5}$ which satisfies the strict inequality in Inequality 6 , since $2=\gamma_{t}\left(K_{5}\right)<\gamma_{t}(\Sigma)=4$.

Corollary 3.8. $\gamma_{t}\left(K_{m, n}^{(r)}\right)=\gamma_{t}\left(K_{m, n}\right)=2$.

Proof. In the case of minimum open dominating set of $K_{m, n}$, it is proved in [2] that $\gamma_{t}\left(K_{m, n}\right)=2$. The vertices being adjacent in the case of open dominating sets, take any one vertex from one part and the other vertex from the next part. This selection will satisfy the condition in Theorem 2.3 and the result follows.

Theorem 3.9. $\gamma_{t}\left(C_{n}^{(r)}\right)=\gamma_{t}\left(C_{n}\right)$ for all $r$ such that $0 \leq r \leq n$. 


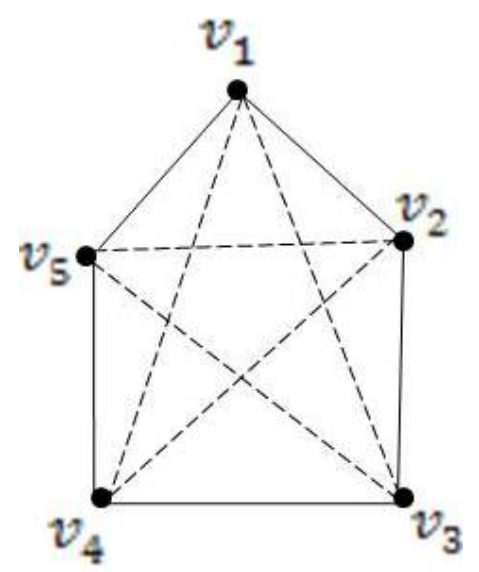

Figure 5: A Signed $K_{5}$

Proof. From Lemma 3.3,it is enough to prove the case for $r=1$. Let $D$ be that minimum open dominating set for $C_{n}$ such that the vertices forming the negative edge belong to $D$ itself. As the marking then depends only on the edges in between $D$ and $V \backslash D$, the all-positive marking will take care of the remaining part of the proof.

In [6], Harary provides an alternative way of looking at balanced signed graphs by giving a partition of the vertex set $V=V_{1} \cup V_{2}$, called Harary's bipartition, one of them possibly empty, such that every negative edge of $\Sigma$ has one of its ends in $V_{1}$ and the other in $V_{2}$ but no positive edge of $\Sigma$ has this property. Regarding the connection between Harary's bipartition and the open domination, we have the following theorem.

Theorem 3.10. If a signed graph $\Sigma=(G, \sigma)$ is balanced and $V=V_{1} \cup V_{2}$ is its Harary bipartition and if $V_{1}$ is an open dominating set for $G$, then it is an open dominating set of $\Sigma$.

Proof. As the negative edges occur only between $V_{1}$ and $V_{2}$ and there are no positive edges between them, we can assign +1 to all vertices in $V_{1}$ and -1 to all vertices in $V_{2}$. This marking will satisfy the definition of open domination.

Acknowledgements. We would like to express our deep sense of gratitude to Professor Thomas Zaslavsky for his incisive and valuable comments on this paper while preparing the same which helped us to immensely improve the content and style of the paper. 


\section{References}

[1] B. D. Acharya, Domination and absorbence in signed graphs and digraphs: I. Foundations. Journal of Combinatorics, Information and System Sciences, To Appear.

[2] Gary Chartrand and Ping Zang, Introduction to graph theory. Tata McGraw-Hill, 2009.

[3] K. A. Germina and Shahul Hameed K, On signed paths, signed cycles and their energies. Applied Mathematical Sciences, 4(70) (2010) 3455 - 3466.

[4] K. A. Germina, Shahul Hameed K and Thomas Zaslavsky, On product and line graphs of signed graphs, their eigenvalues and energy. Linear Algebra and its Appl., 435 (2011) 2432-2450.

[5] F. Harary, Graph Theory. Addison Wesley, Reading, Massachusetts, 1972.

[6] F. Harary, On the notion of balance of a signed graph. Michigan Math. J., 2 (1953) 143-146.

[7] T. Haynes, S.T. Hedetneimi and P J Slater, Fundamentals of Domination in Graphs, Marcel Dekker, 1998.

[8] T. Zaslavsky, Signed graphs. Discrete Appl. Math. 4 (1982) 47-74. Erratum. Discrete Appl. Math. 5 (1983) 248.

[9] T. Zaslavsky, A mathematical bibliography of signed and gain graphs and allied areas. VII Edition, Electronic J. Combinatorics, 8(1998), Dynamic Surveys, \#8, 124 pp.

\section{Received: November 1, 2013}

